\title{
Tomographic X-ray particle tracking velocimetry
}

\section{Proof-of-concept in a creeping flow}

\author{
Simo A. Mäkiharju ${ }^{1}\left[\right.$ ] Jan Dewanckele ${ }^{2} \cdot$ Marijn Boone $^{2} \cdot$ Christian Wagner $^{3} \cdot$ Andreas Griesser $^{3}$
}

Received: 13 April 2021 / Revised: 30 October 2021 / Accepted: 27 November 2021 / Published online: 23 December 2021

(c) The Author(s) 2021

\section{Abstract}

We investigate the feasibility of in-laboratory tomographic X-ray particle tracking velocimetry (TXPTV) and consider creeping flows with nearly density matched flow tracers. Specifically, in these proof-of-concept experiments we examined a Poiseuille flow, flow through porous media and a multiphase flow with a Taylor bubble. For a full $360^{\circ}$ computed tomography $(\mathrm{CT})$ scan we show that the specially selected 60 micron tracer particles could be imaged in less than 3 seconds with a signal-to-noise ratio between the tracers and the fluid of 2.5 , sufficient to achieve proper volumetric segmentation at each time step. In the pipe flow, continuous Lagrangian particle trajectories were obtained, after which all the standard techniques used for PTV or PIV (taken at visible wave lengths) could also be employed for TXPTV data. And, with TXPTV we can examine flows inaccessible with visible wave lengths due to opaque media or numerous refractive interfaces. In the case of opaque porous media we were able to observe material accumulation and pore clogging, and for flow with Taylor bubble we can trace the particles and hence obtain velocities in the liquid film between the wall and bubble, with thickness of liquid film itself also simultaneously obtained from the volumetric reconstruction after segmentation. While improvements in scan speed are anticipated due to continuing improvements in CT system components, we show that for the flows examined even the presently available CT systems could yield quantitative flow data with the primary limitation being the quality of available flow tracers.

\section{Graphic abstract}
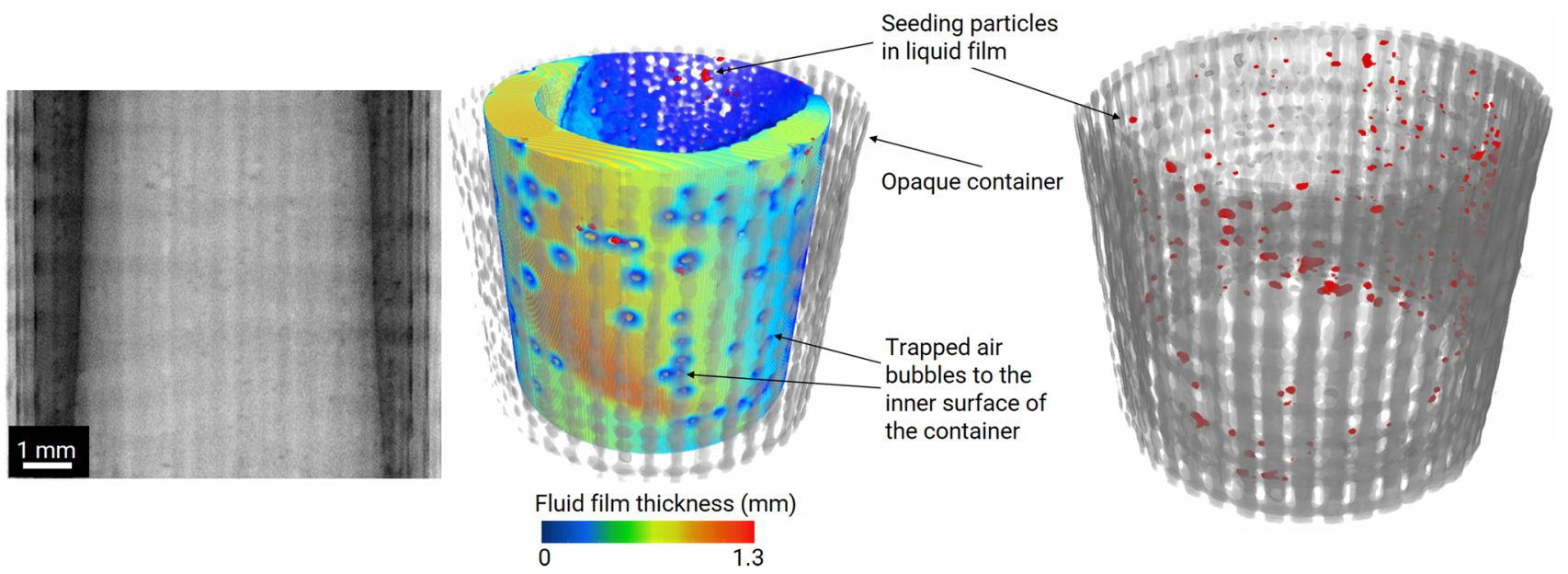

Simo A. Mäkiharju

makiharju@berkeley.edu

Extended author information available on the last page of the article 


\section{Introduction}

Multiphase flows are all around us, and inside us. From waves breaking and exchanging atmospheric gases with the ocean water, to the blood in our veins with red blood cells in plasma. Fundamental discoveries in these flows lead to energy savings, medical advancements and reduced pollution. And much remains to be learned. For example, only recently, it was discovered that under certain conditions shedding cavitation in an adverse pressure gradient can be dominated by shock waves instead of re-entrant jets (Ganesh et al. 2016; Mäkiharju et al. 2017). Discoveries such as these are enabled by new "in-the-lab" quantitative measurement techniques, some utilizing X-ray techniques (e.g., Mäkiharju et al. (2013)). Some of the information we need to be able to obtain with improved or new experimental techniques include: phase fractions and velocity fields in highly thermally conducting (e.g., metal) foam filled channels and porous media, flow velocities of multiple phases even in annular flows, and flow velocities in cavitating and other opaque flows.

Particle Image Velocimetry (PIV) (Raffel et al. 2018) with visible light is an extremely useful experimental technique for single phase flows. However, due to the opaque nature of most multiphase flows, use of PIV for them has been limited. If we can go beyond the visible spectrum, photons in the X-ray range have the advantage of having refractive indices near unity (Attwood and Sakdinawat 2017). Hence, X-ray PIV (XPIV) has been a topic of interest and non-tomographic XPIV has been demonstrated using synchrotron X-ray sources (Lee and Kim 2003). 2D X-ray PTV was also shown feasible at a synchrotron where microbubbles where simultaneously sized and tracked (Lee and Kim 2005). And, a type of TXPTV at a synchrotron was first shown feasible by (Im et al. 2007), who collected 240 projections in 30.6 seconds, and subsequently reconstructed a 3D velocity field from 2D velocity field projections. Subsequently, improved synchrotron TXPIV was conducted by Dubsky et al. (2010) with a 10 second scan time. However, synchrotrons are not readily accessible to all researchers and not usually available for extended periods of time. Bringing 2D XPIV /XPTV, and even tomographic XPIV (TXPIV) or TXPTV, to the laboratory scale is of great interest. The few and recent early applications of in-the-laboratory XPIV utilized $O(1 \mathrm{~mm})$ tracers (Smith et al. 2018). The large particles may not faithfully follow most flows of interest due to their Stokes number. One may note that in PIV the typical seeding particle has a diameter between 1 and $100 \mu \mathrm{m}$. However, particles in this range with composition suitable to yield usable contrast when used with in-laboratory X-ray sources and detectors are not easy to come by or manufacture.
We identified suitable tracers (Parker and Mäkiharju 2021) and examine the feasibility, of in-the-laboratory TXPTV with $60 \mu \mathrm{m}$ silver-coated particles utilizing a stateof-art micro-CT scanner. While these first flows studied are creeping flows, one may later extend the approach to faster flows as the tracers (Parker and Mäkiharju 2021), source brightness and detector performance are continuing to improve. Indeed, X-ray sources such as the Metaljet series (Espes et al. 2015) alone promise to increase in-laboratory achievable source brightness by two orders of magnitude. Coupled with faster more sensitive detectors, such as photon counting energy thresholded detector arrays, XPIV/XPTV and TXPIV/TXPTV can gain increasing value as research techniques. The present study is a proof-of-concept of TXPTV examining the techniques that once developed for creeping flows are also employable to increasing number of flows, as faster in-laboratory XPIV/XPTV and TXPIV/ TXPTV become practical.

In Sect. 2 we provide a description of the experimental setup and methods. Results from the proof-of-concept pipe flow experiment, porous media flow and flow with Taylor bubble are provided in Sect. 3, followed by the conclusions in Sect. 4.

\section{Experimental setup and methods}

$\mathrm{X}$-ray Computed Tomography, is a technique used to reconstruct a $3 \mathrm{D}$ object from a series of $2 \mathrm{D}$ projections. The advantage of CT for flow measurements is that X-rays can penetrate visually opaque confinements, and at wavelengths used refractive indices are close to unity (Attwood and Sakdinawat 2017). The X-ray attenuation, following Beer-Lambert law, is measured in the projection direction and is proportional to the pixel intensity on the radiograph. And, owing to the development of faster CT scanners, such as the one utilized, combined with suitable flow tracers, we can begin to utilize these tools traditionally used for non-destructive testing of static objects to dynamic objects and flows.

The experiments were conducted with a TESCAN CoreTOM micro-CT system (Fig. 1). A tube voltage of $60 \mathrm{kV}$ and a power of $25 \mathrm{~W}$ was utilized. The flow was confined inside an optically opaque, but minimally X-ray attenuating, carbon fiber tube with an inner diameter of $6.35 \mathrm{~mm}$, and wall thickness of $1 \mathrm{~mm}$. The center of the tube containing the flow was positioned $15 \mathrm{~mm}$ from the X-ray source, and with detector $400 \mathrm{~mm}$ away. This given the Pixel pitch $0.45 \mathrm{~mm}(0.150 \mathrm{~mm}$ natively on detector plane, but scanned in Binning mode resulted in a reconstructed voxel size of $17 \mu \mathrm{m}$. This voxel dimension was found to be adequate to 


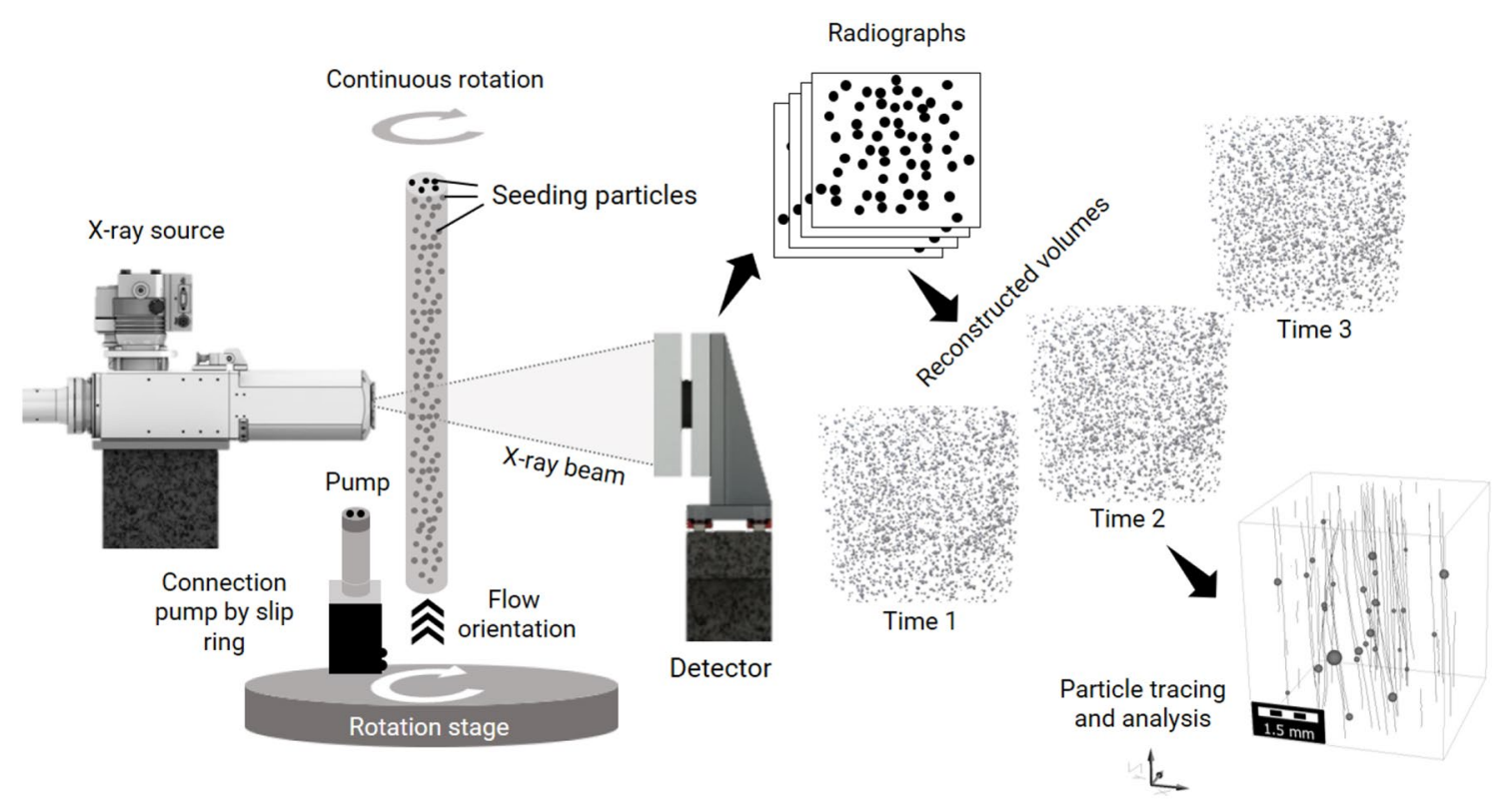

Fig. 1 Overview of the experimental micro-CT set-up. Fast, continuous rotation of the in situ set-up enabled dynamic reconstruction and tracing of the individual seeding particles. Subsequently, the particle locations were identified and trajectories reconstructed as discussed in Sect. 2.3

localize the individual seeding particles used. A mixture of glycerol (70\%) and water (30\%) was used as the fluid and seeded with $\approx 60 \mu \mathrm{m}$ diameter hollow, silver-coated glass spheres described in Sect. 2.1. The mixture was pumped through the optically opaque tube at a flow rate of $\approx 20 \mu \mathrm{ll}$ min, using a M6 series Syringe-Free Liquid Handling pump (VICI) with flow rate adjustable from $5 \mathrm{nl}$ to $5 \mathrm{ml} / \mathrm{min}$, with manufacturer specified nominal volume precision (\%CV) of $<0.1 \%$ at $1.25 \mathrm{ml}$ and $<0.5 \%$ at $125 \mu l$.

The complete in situ set-up was mounted on the rotation stage of the TESCAN CoreTOM and the pump was fed through the slip-ring utilizing the in situ integration kit of the system. This enabled us to continuously rotate the tube over multiple turns at rate of 120 degrees/second during the experiment (Fig. 1). As the particles were nominally matched to density of water, and the centripetal acceleration even for a particle at the wall of the tube was only $\approx 0.14 \%$ of gravitational acceleration, $g$, the rotation should not to first order influence the particle trajectories, which is consistent with the observed particle behavior (see Sect. 3.1).

A single rotation $\left(0-360^{\circ}\right)$ scan time of less than 3 seconds was achieved through the use of a high-speed silicon flat panel detector operated at a frame rate of $68 \mathrm{fps}$ (with exposure time of $14.5 \mathrm{~ms} /$ projections). The sample was rotated over $72,000^{\circ}$ (200 continuous full $360^{\circ}$ rotations), while taking 200 radiographs for each full rotation. To perform the reconstruction of the acquired dynamic process, the FDK algorithm was used as a natural 3D extension of the filtered-back projection algorithm (Kak and Slaney 1988; Feldkamp et al. 1984). As a result, a 3D array of voxels can be reconstructed. In total, for each proof-of-concept experiment 200 reconstructed volumes with $480 \times 480 \times 344$ voxels were acquired. The total experiment time for each experiment was $11 \mathrm{~min}$, including taking open-beam (flat field) and dark field images used for normalization. The sample to detector distance was optimized to achieve maximum signal and resolution and an optimum of $400 \mathrm{~mm}$ was found to provide the best image quality in present experiment. Here the signal-tonoise ratio (SNR) is defined as

$S N R=\frac{m_{\text {particles }}-m_{\text {fluid }}}{\frac{1}{2}\left(\sigma_{\text {particles }}+\sigma_{\text {fluid }}\right)}$

and it was calculated to be 2.5 between the tracer particles and the fluid. Here $m=$ mean and $\sigma=$ standard deviation, in regions of the image identified by the subscript.

We also note that in the $14.5 \mathrm{~ms}$ it takes to collect a single exposure an image of a particle on the interior pipe wall in the section closest to or furthest from the source could be smeared across nearly six pixels. However, the CT reconstruction still recovered a sharp and easy to localize particle, as during a full rotation for an appreciable fraction of time the same particle on the wall will appear sharp (when the particle is moving nearly normal to the imager plane). Sources of blur and motion artifacts are further discussed in the appendix. 


\subsection{Choice of particles}

An ideal tracer must be (at least nominally) neutrally buoyant and small enough that in given flow its Stokes number enables it to follow the flow with high fidelity (Raffel et al. 2018). However, for TXPTV as an requirement different from PTV and PIV at visible wavelengths, it must incorporate material with X-ray attenuation in relevant range of photon energies which is substantially higher or lower than attenuation due to surrounding fluid(s). While under certain circumstances some of the standard PIV tracers may be suitable for XPIV or TXPTV, this is not the case in general as discussed by Parker and Mäkiharju (2021), who present a systematic theoretical and experimental study relevant for XPTV flow tracer selection and development.

For the present study we utilized silver-coated hollow glass beads, AGSF33, from Potters Beads with nominal 50 micron average diameter and a density of $0.9-1.1 \mathrm{~g} / \mathrm{cc}$. However, as the particles were not perfectly monodisperse, they were professionally sieved and particles from range of 53-75 $\mu \mathrm{m}$ utilized, nominally having a mean diameter of $60 \mu \mathrm{m}$. After sieving, the particles were also pre-filtered (floated) to remove the density outliers that either sunk or rose to the top in fluid used in less than 4 minutes. (However, as will be discussed in Sect. 3.1, it would be beneficial if the particle density were even better matched to that of the fluid unless a mixture of higher viscosity were used, or a faster flow investigated.)

\subsection{Choice of fluid}

For the fluid we used a mixture of glycerol (70\% by mass) and water (30\% by mass) at $20^{\circ} \mathrm{C}$, which yields a higher viscosity than pure water, as our goal was to have a low Reynolds number creeping flow.
The fluid density was calculated based on formula of (Volk and Kähler 2018), who also consider volume contraction, and found to be $1181 \mathrm{~kg} / \mathrm{m}^{3}$. Based on the mixture mass fractions, temperature and equation of Cheng (2008), the fluid's kinematic viscosity is $v=19.6 \times 10^{-6} \mathrm{~m}^{2} / \mathrm{s}$, and dynamic viscosity, $\mu$, of $0.023 \mathrm{Ns} / \mathrm{m}^{2}$.

\subsection{Particle localization and tracking}

Following steps were sufficient to convert the CT scans into particle trajectories. First, CT reconstructions gray-value noise was reduced utilizing the Non-Local Means filter (Buades et al. 2005). Second, Otsu's segmentation method (Otsu 1979) was used to distinguished the particles from the liquid and outer regions. Figure 2 shows single slice from the original, filtered and segmented 3D volume.

After the segmentation, individual particles had to be identified, Fig. 3a. Since particles can be clumped together in small clusters, clusters larger than a single particle were subdivided to recover the individual particles. This was achieved by applying a watershed-based (Vincent and Soille 1991; Borgefors 1984) algorithm on each of the particle clusters. In the resulting 3D volume every voxel is assigned a unique particle id.

To be able to track the particles, their centroids were required, and notably as only the centroids are tracked were a reconstructed particle to appear elliptical due to motion blur this would not impair the PTV. To find the centroid, an ellipsoid was fitted to the set of voxels belonging to the same particle (having same particle id) (Pearson 1901). Finally, particle tracing over time is achieved by matching closest particles centroids in consecutive time steps, see Fig. 3 b. (We may note that had the flow been more densely seeded or motion been greater during individual time steps, a more
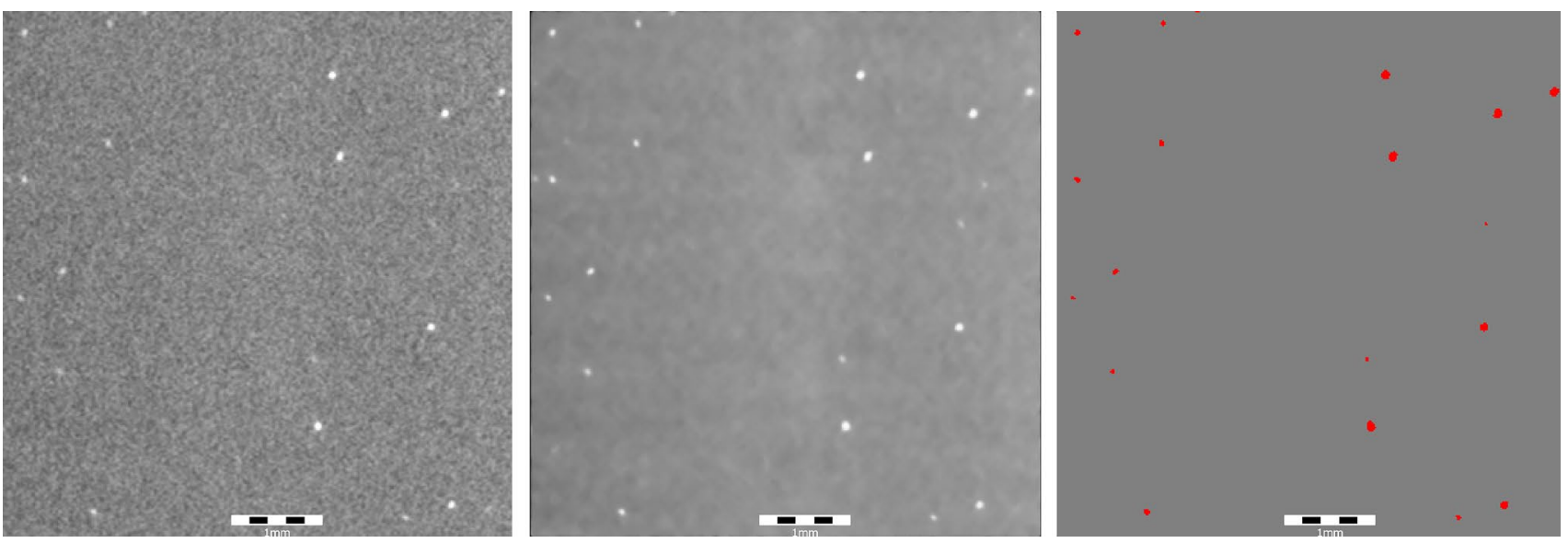

Fig. 2 Comparison of one slice from the 3D reconstructed volume with the initial gray values (left), noise-filtered image of same slice (middle), and segmented image (right) 
(a)

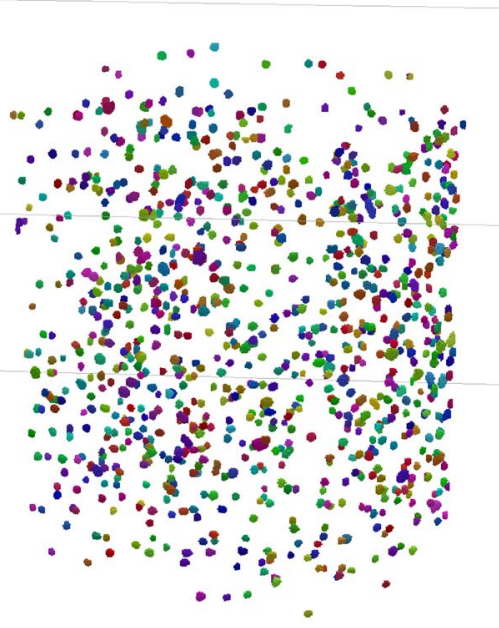

$1 \mathrm{~mm}$

(b)

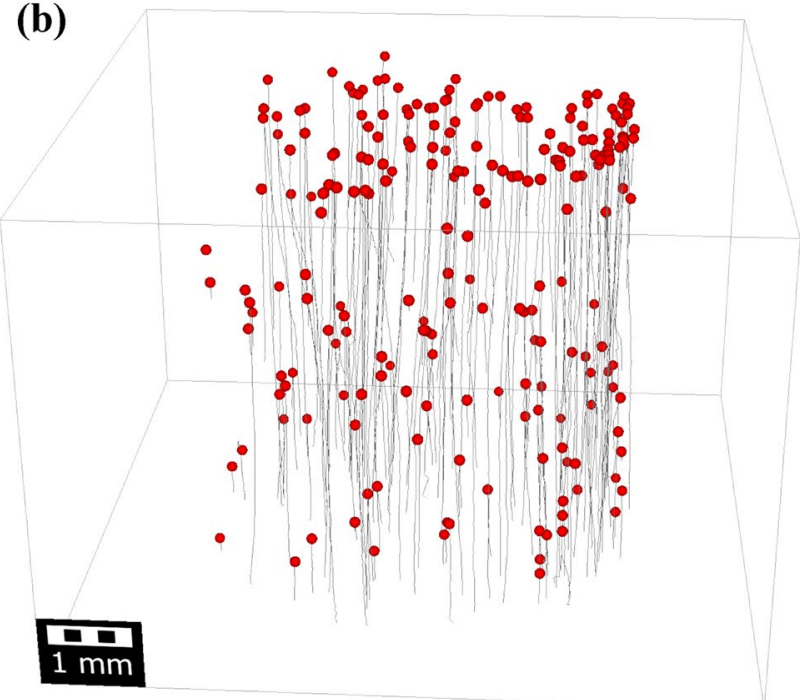

Fig. 3 a Segmented image where individual particles are identified by different colors. b Particle trajectories on a subset of the particles

advanced cost function based approach to match the particles could have been necessary.)

\section{Results}

\subsection{Poiseuille flow}

The flow loop consisted of a $6.35 \mathrm{~mm}$ inner diameter pipe (1/4") through which fluid was pumped at $20 \mu \mathrm{l} / \mathrm{min}$. Hence, the average velocity is expected to be $10.5 \mu \mathrm{m} / \mathrm{s}$. Subsequently, our Reynolds number based on pipe inner diameter, $R e=U d / v$, is $\approx 0.0034$. Thus, neglecting the effect of particles due to low solids fraction $(\approx 0.1 \%)$, we expect entrance length for flow development to be $L_{e} / d \approx<1$ (Atkinson et al.
1969) for $\operatorname{Re}<0.1$, as is our case. Hence, from the TXPTV expect to find a parabolic velocity profile based on the Navier-Stokes equations. This classic solution, Poiseuille flow, is given by

$\frac{u(r)}{2 \bar{U}}=1-\left(\frac{r}{R}\right)^{2}$

where $\bar{U}$ is the average velocity, $u(r)$ the velocity as a function of radial coordinate from pipe center and $R$ is the inner pipe diameter.

Figure 4 shows the comparison of the average flow field based on TXPTV with dilute particle concentration (such that flow can be presumed one way coupled) and theoretical flow profile of Eq. 2 should hold. We notice that the profile appears shifted and there are numerous outliers (i.e., some individual particles had velocities deviating from what was expected given their radial coordinate). Given that the mean velocity was mere $10.5 \mu \mathrm{m} / \mathrm{s}$, this, however, is not surprising. If our 60 micron particle's density deviated from fluid density the slightest, and using Stokes drag with drag coefficient given by $C_{D}=24 / R e$ we readily find the terminal velocity (velocity relative to fluid velocity) resulting from a small density difference. In our 70/30 glycerol/water mixture, for a 5 or $15 \%$ density difference, the particle velocity with respect to terminal velocity will be 5 or $13 \mu \mathrm{m} / \mathrm{s}$. Or, more generally, as long as Reynolds number based on

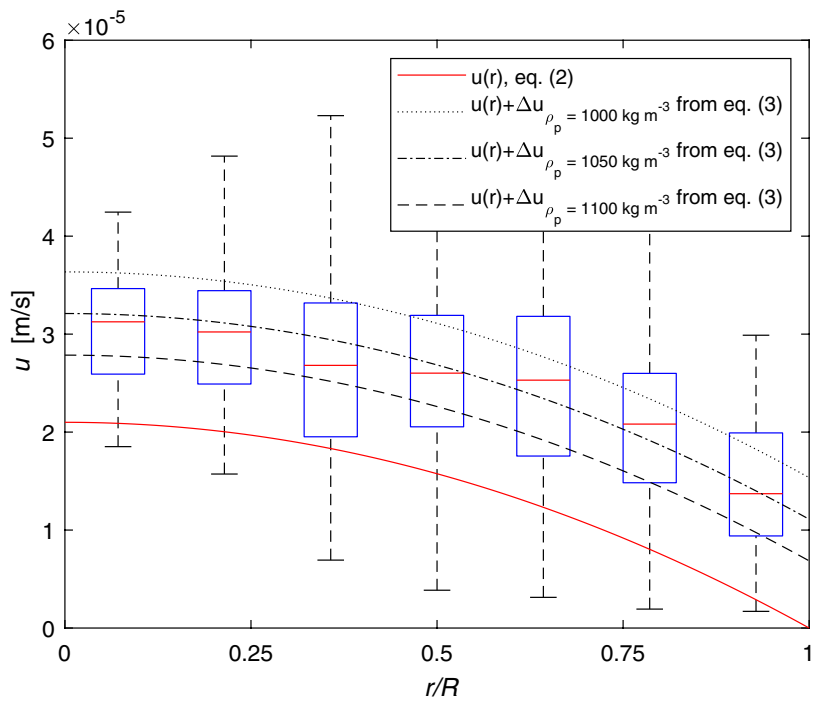

Fig. 4 Comparison of velocity profiles measured by TXPTV and theoretical Eq. 2 (red line). However, if we account for density difference of fluid and particles based on Eq. 3 for particles that are 1000, 1050 or $1100 \mathrm{~kg} / \mathrm{m}^{3}$ we would expect the velocity shown by the dotted, dash-dot and dashed curves, respectively. Each box represents the statistics of the particles within $r \pm \Delta r_{b i n} / 2$, and the red line shows the median with the top and bottom of the box indicating $75^{\text {th }}$ and $25^{\text {th }}$ percentiles, respectively. The whiskers indicate full range of data, excluding outliers ( $>2.7 \sigma$ away from median) 
terminal velocity is less than unity, we can estimate terminal slip velocity in direction of gravity due to density deviation $\phi_{\rho}=\rho_{\text {particle }} / \rho_{\text {fluid }}$ as

$\Delta u_{\text {buoyancy }}=\frac{2 g r_{\text {particle }}^{2}\left(\phi_{\rho}-1\right)}{9 v_{\text {fluid }}}$

The effects of the imperfect density match are further discussed in Parker and Mäkiharju (2021).

Hence, we can also observe that the scatter in velocity could be due to variation of particle density in the $3 \%$ range. This observation highlights that while the chosen particles provided better contrast than other particles examined, these silver-coated hollow glass spheres still likely had too much variation in their average density to be ideal for investigating such a slow creeping flow. Indeed, in present case for particle velocity to be within, e.g., $5 \%$ of center line velocity, we should have matched fluid density within $1 \%$. While technically achievable, such particles were not available for present work.

However, even with these imperfectly density matched particles had

1. the source brightness and detector enabled a 20x faster acquisition, velocity error due to density mismatch would have been within $5 \%$, or

2. had we been able to use a more viscous fluid, such as $100 \%$ glycerol we would have expected a $\Delta u_{\text {buoyancy }}<1 \mu \mathrm{m} / \mathrm{s}$. (In the present experiments use of higher viscosity fluid was inhibited by limitations of the pump available.)

As limiting use of in-laboratory TXPTV to high viscosity fluids is undesirable, clearly either $i$ ) brighter in-laboratory $\mathrm{X}$-ray sources and faster detectors are necessary, such as one might anticipate to became available this decade, or $i i$ ) one needs better density matched tracers (ideally with higher $\mathrm{X}$-ray contrast such as those proposed by Parker and Mäkiharju (2021) and presently under development).

Next we consider if, with CT systems and particles presently available, TXPTV can be utilized for flow in porous media and in two phase flows-with increasing fidelity if better tracers were to become available.

\subsection{Micron-scale particle migration and retention in $3 \mathrm{D}$ porous media}

Understanding flow dynamics in porous materials such as porous rocks is essential for many applications including ground water resources, oil and gas, $\mathrm{CO}_{2}$ sequestration, geothermal energy and storage of nuclear waste. Intrinsic parameters such as porosity, pore size distribution, connectivity, surface roughness and area, etc. will have a significant impact on the transport properties and thus the flow path through the material. In addition, the changing properties, such as the migration and retention of fine particles (e.g., clay particles) will play a central role in clogging and thus overall transport properties (Liu et al. 2019).

For this experiment, a $65 \mathrm{~mm}$ tall pipe with $6.35 \mathrm{~mm}$ ID with a sintered glass sample filling bottom $15 \mathrm{~mm}$ of the pipe was scanned. The flow resulted from drainage through a $0.5 \mathrm{~mm}$ ID $750 \mathrm{~mm}$ long pipe driven by a pressure gradient due to an $800 \mathrm{~mm}$ elevation difference. With 70/30 glycerol/water mixture, ignoring minor losses and losses in porous media as first approximation, based on pressure drop due to laminar flow in the $0.5 \mathrm{~mm}$ ID pipe this would have resulted nominally in a $26 \mu \mathrm{m} / \mathrm{s}$ flow in the $6.35 \mathrm{~mm}$ ID section of tube. If we assume pore fraction was $25 \%$ a flow speed $O(100 \mu \mathrm{m} / \mathrm{s})$ would have resulted. Hence, we assume as first approximation the effect of gravitational settling with terminal velocity $O(10 \mu \mathrm{m} / \mathrm{s})$ can be neglected and expect to only resolve particles that have gotten stuck, thus clogging pores, or that are in a low flow region, if one exists. To verify this assumption we simulated the flow through the sintered glass section of the pipe as it was at the beginning of the experiment without any pores clogged. The simulation was performed using the LIR solver (Linden et al. 2015) in GeoDict. The results, shown in Fig 5, confirmed the expected high flow velocities in the pores, which explained why in the present experiment it was not possible to image particles until they were captured by constrictions or caught in pockets with low flow velocities.

The same acquisition parameters were use as described previously in Sect. refsec:exp. In total, 8 dynamic experiments (each 200 rotations, $<3$ s/rotation) were performed with an interval time of $\approx 10 \mathrm{~min}$ in between. The voxel size was $18 \mu m$.

During one single dynamic experiment it was possible to investigate the clogging of the pores by the particles by using a flippoint detection. The flippoint detection is an automated detection of those voxels that are changing during the experiment. Voxels of the glass structure will not change gray value during the experiment, and will be referred as stable voxels. However, in the pore structure fluid migrates with tracer particles, thus changing from the initial voxel gray value through time (dynamic voxels). Instead of analyzing all the reconstructed 3D volumes individually, a time dimension is incorporated by analyzing the gray value. The time attributed to an event corresponds with the time when a voxel exceeds the threshold (to exclude effects of noise) of a changing gray value. In this case, when a particle is trapped in a pore neck, the gray value will change from gray (pure fluid) to white (trapped particle). That specific voxel location will be relabeled as a time stamp instead of an absorption value. As can be seen on Fig. 6, a blocking seeding particle (white) is visible (in left most figure). The seeding particles in the fluid 
(a)

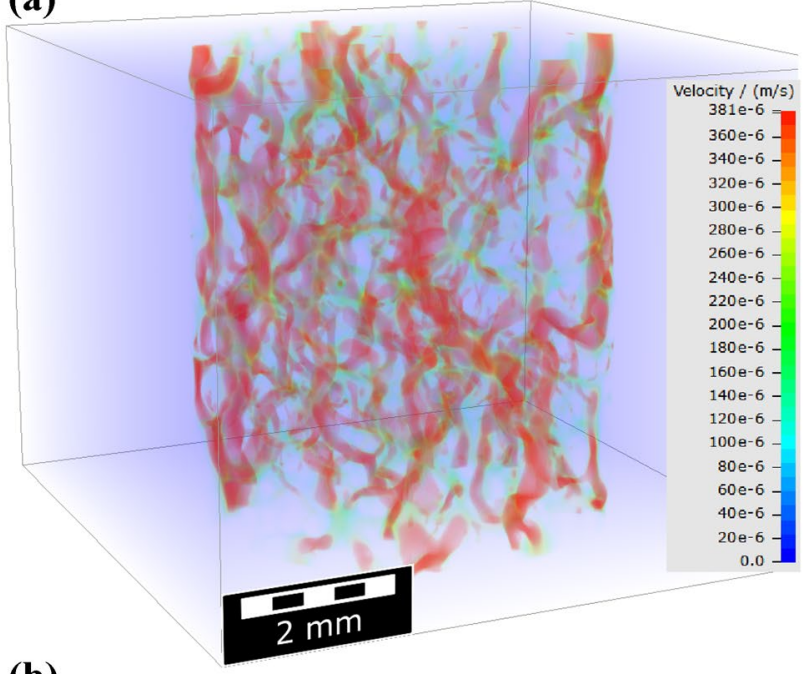

(b)

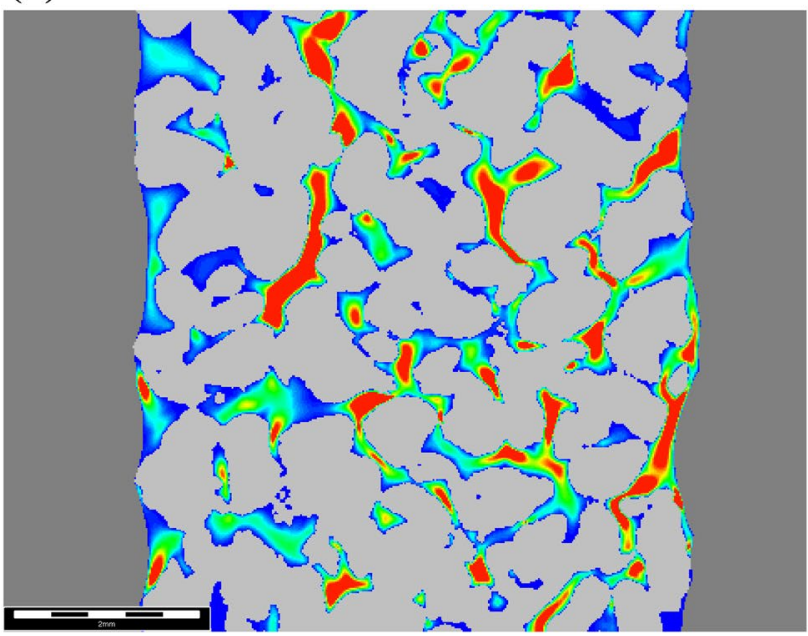

Fig. 5 a 3D view of the simulated flow through sintered glass structure, which was reconstructed based on the CT scan. b 2D slice of the structure and flow field in the pores. Blue corresponds to low flow velocities and red to high flow velocities up to $381 \mu \mathrm{m} / \mathrm{s}$

itself are not resolved due to the nominal flow speeds being $O(100 \mu \mathrm{m} / \mathrm{s})$ in the pore structure. But, once a seeding particle (or cluster of them) gets stuck it becomes visible and detectable in the reconstructed volume. By applying the flippoint detection, the change inside the volume is pinpointed both in time and space. As can be seen in Fig. 6 (flippoint detected; middle) the clogged particle displays now a clear time point when it got retained (at $130 \mathrm{~s}$ after the start of the experiment). On the other hand, flippoints are also detected in most of the open pore structure, meaning that some changes in grey value have taken place there as well, corresponding to the movement of the fluid and tracer particles. So, although we are not able to detect the individual moving particles in the fluid, they might contribute to the detection of the flippoint in the pore structure. When rendering the results of the flippoint (6; right), the clogging process in both space and time (color) becomes visible, starting from the top of the sample (inlet of the flow), enabling the understanding of the migration patterns.

On the other hand, taking all the dynamic results into account, a complete overview of the process becomes visible as can be seen on Fig. 7, demonstrating a vertical cross section before and after the experiment. A segmentation of the clogged particles can be performed on the end state of each dynamic experiment. By doing so, the evolution of the clogging can be visualized for individual pore structures on micron-scale (see Fig. 8) .

\subsection{Taylor bubble and flow around it}

As a slug of gas (i.e., Taylor bubble) forms in a multiphase flow and travels along a pipe, there is still a liquid film between the wall and the bubble. If in this film we can trace particles, we can measure velocity withing the film. While for flow over a smooth hydrophilic surface one may find solution to be obtainable analytically, measurement of the velocity in such a film may yield a non-trivial result if instead of a solid wall we had, e.g., a structured or hydrophobic surface with second phase present (trapped gas).

We found that the film between a Taylor bubble and the pipe wall could be resolved in the same experimental setup with the same flow and acquisition parameters as used for Sect. 3.1. In the present experiment, it was possible to observe a Taylor bubble in 3D. In this case, the speed of the Taylor bubble is around $5 \mathrm{~mm} / \mathrm{s}$. With the present spatial and temporal resolution, the Taylor bubble needed to be at least 15 $\mathrm{mm}$ long in order to fully resolve the body of the bubble and the liquid film. For shorter bubbles, the head and or tail of the bubble may cause movement artifacts during the reconstruction. If the body is completely resolved within the temporal resolution, it is possible to calculate the thickness of the liquid film around the bubble. As shown in this example (Fig. 9), we also resolve the smaller 150-200 $\mu \mathrm{m}$ bubbles trapped in the liquid film and attached to the inner surface of the tube (blue circles), and the tracer particles (shows as red dots). We observed in this particular case with bubbles trapped on the walls that the liquid film demonstrates a non-uniform distribution with a thicker film on one side of the bubbles stuck to the wall. Increasing scan speeds would enable more detailed measurement of the flow around the ends of the bubble as well, while simultaneously resolving dispersed bubbles that may be present and particle tracer trajectories.

\section{Conclusions and future direction}

The feasibility of using a state-of-art fast CT scanner for inthe-laboratory tomographic X-ray particle tracking velocimetry (TXPTV) was investigated. For the first time we 

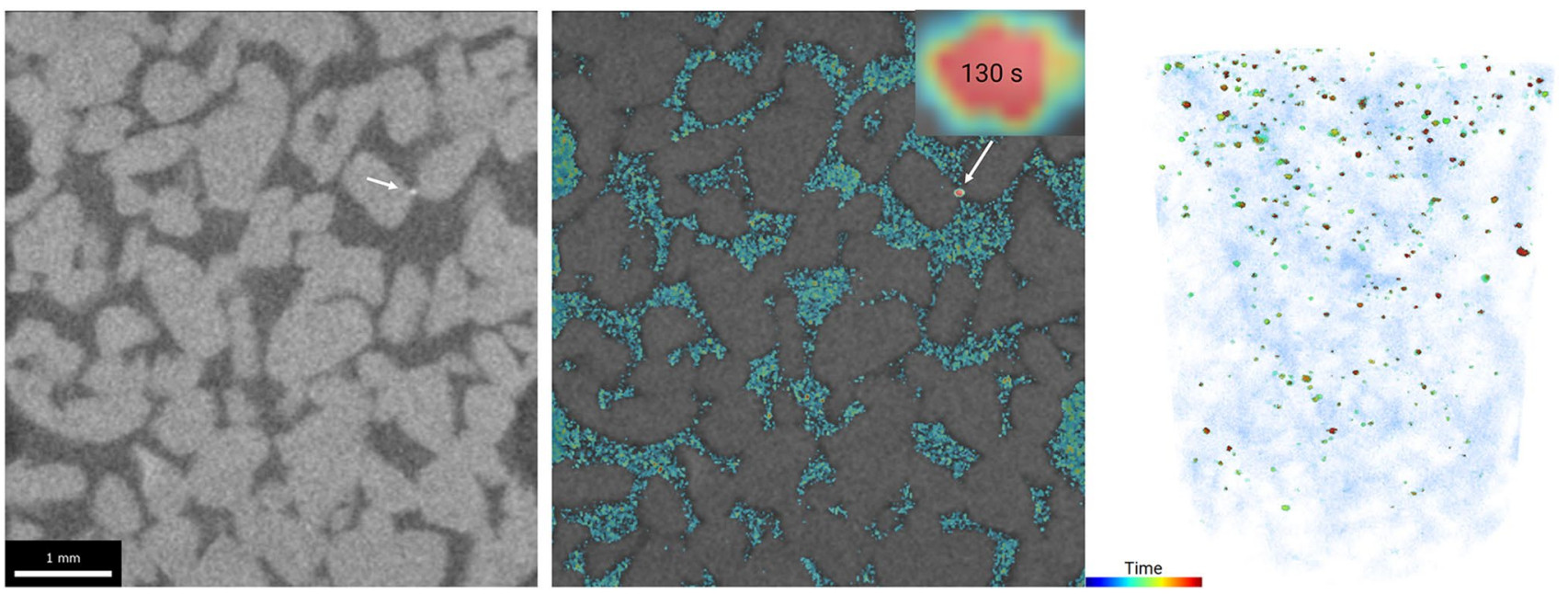

Fig. 6 Cross section during the first dynamic experiment showing the sintered glass beads and particle laden fluid in the pore space (left); detection of the flippoint, indicating the changes in grey values at a certain time interval (middle) and 3D render of the complete sample showing the position and timepoints (color) of the clogging seeding particles
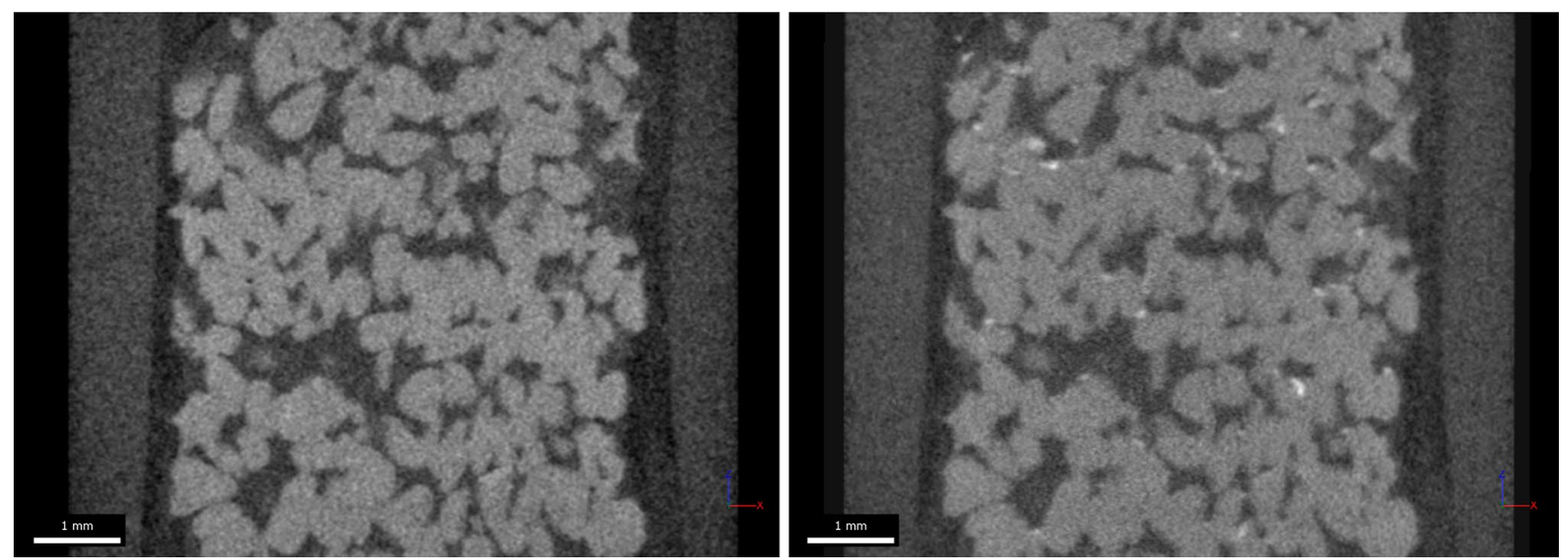

Fig. 7 Vertical cross section through the sintered glass sample reconstructed from a $<3$ second $360^{\circ}$ scan. (Left) the beginning of the experiment and (right) the end state. The bright spots in the end state correspond to the seeding particles clogged in the pores

show the feasibility of i) in-laboratory Tomographic XPTV (which can be much more widely available than synchrotron TXPTV), and ii) in-laboratory TXPTV with sub mm (actually sub $100 \mu \mathrm{m}$ ) particles. Full CT reconstructions with sufficient resolution and contrast to localize both gas-liquid phase boundaries and $60 \mu \mathrm{m}$ particles at scan rate of $3 \mathrm{sec}-$ onds were found to enable tracing of individual particles in the flows considered. In porous media, clogging of individual pores as a function of time could be observed, and for the Taylor bubble we were able to measure the thickness of the film, which was greater on side of the tube that had dispersed bubbles trapped on the wall, and localize seeding particles.

For the purposes of TXPTV one could define as success a case where particles had sufficient contrast to be detected in the first place while being nominally neutrally buoyant, and particle centroids are localizable in the CT reconstruction. However, while feasibility and potential of in-laboratory TXPIV utilizing a state-of-art micro-CT scanner was shown, by a stricter standard this first proof-of-concept data presents only a partial success. Imperfections of the flow tracers that were available were found to limit what is presently achievable with in-laboratory TXPTV. Specifically, while in a creeping flow a Poiseuille flow profile could be qualitatively observed, it was evident that the tracer particles utilized were not sufficiently uniform in density (nor well matched in density to that of the fluid) to allow high fidelity measurement of such low flow speeds, unless fluid viscosity could have been increased further. For the porous media case 


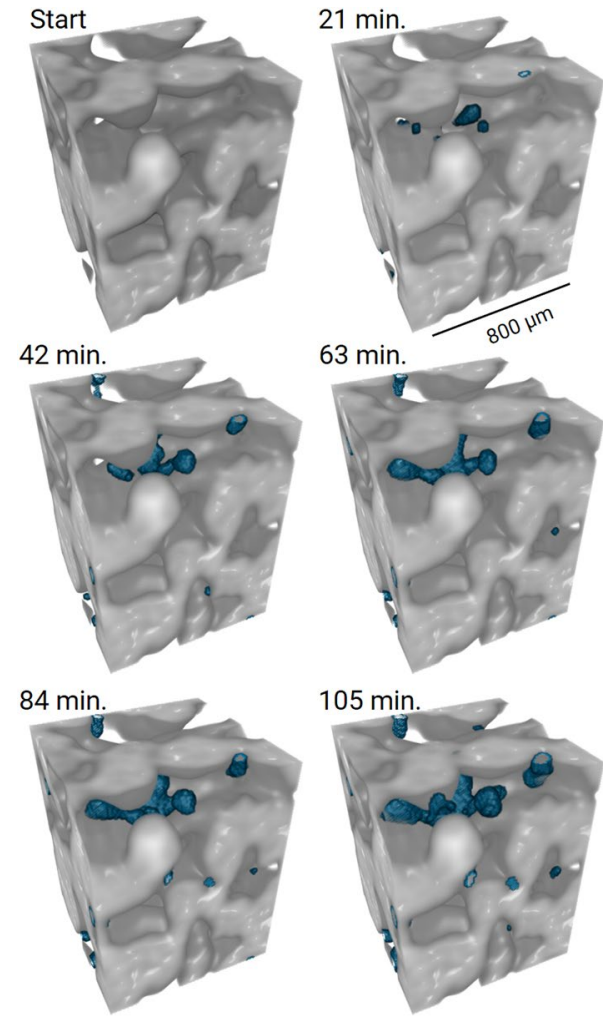

Fig. 8 Time-series of an individual pore clogging due to the accumulation of particles (blue)

and Taylor bubble, to observe the flow directly improvement in scan speed of 10x or tracer particles with better density match would have also been necessary to enable such observation, and this will likely be feasible in the near-term.

While at the present scan rates, even with better tracer particles, in-laboratory TXPTV applicability may be limited to creeping flows, as with film based PIV in the 80s and 90s, the TXPTV techniques developed for these slow flows are readily transferable to higher speed applications of increasing importance. This motivated the present study, beyond the applications already accessible with the demonstrated scan speeds. Furthermore, our ongoing work to advance TXPTV/ TXPIV includes design and examination of particles with tighter range of density (such as discussed in Parker and Mäkiharju (2021)), and utilization of sparse angle or limited angle CT data to enable faster acquisition even with present source brightness limitations, and employing brighter in-laboratory X-ray sources as they become more widely available (Espes et al. 2015). The detailed exploration of algorithms best suited for TXPIV requires additional study. Iterative algorithms, such as (Yoon et al. 2017), may further decrease the particle localization uncertainty with limited number of projections. Indeed, we may note that particles themselves could have been localized from a fraction of the projection angles. However, the benefit of the full tomographic X-ray PTV/PIV is that the flow domain geometry, which may be unknown or time varying, can also be reconstructed. Such was the case in the present study when we considered flow in porous media and Taylor bubbles in a pipe with secondary microscopic dispersed bubbles. Clearly, TXPTV will be increasingly usable as laboratory X-ray source and detector technologies inevitably improve.

\section{Appendix: Motion artifacts}

For the present experiments each X-ray image exposure was $14.5 \mathrm{~ms}$ long and due to the flow the particles were moving at an average velocity of $\approx 20 \mu \mathrm{m} / \mathrm{s}$ (with few reaching speeds up to $50 \mu \mathrm{m} / \mathrm{s}$ ). As the particle diameter was $60 \mu \mathrm{m}$,
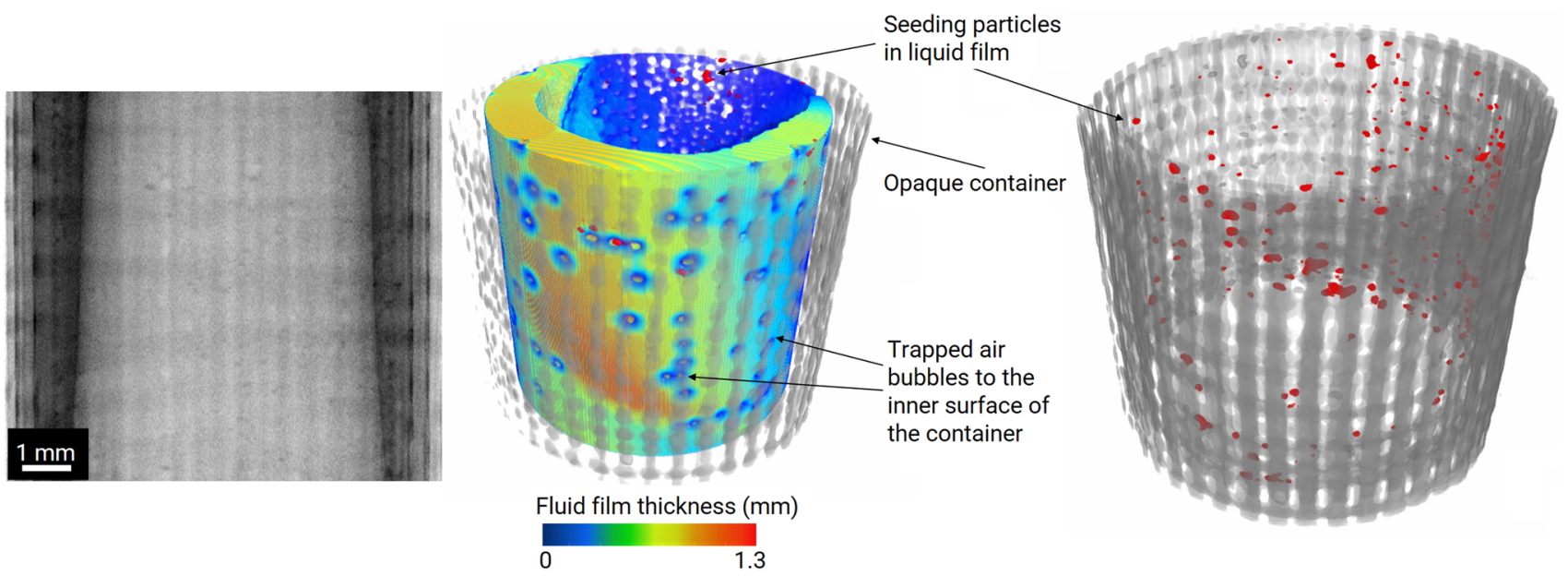

Fig. 9 Projection image of Taylor bubble (left); rendered wall thickness of the Taylor bubble in 3D and dispersed gas bubbles trapped on the wall (middle); seeding particles shown in red withing the liquid film (middle and right) 
during a single exposure motion blur due to flow would be limited to $\approx 0.3 \mu \mathrm{m}$ for the average particle and $0.7 \mu \mathrm{m}$ (or $1.2 \%$ of particle diameter) for the fastest particles. Hence the individual exposures had negligible motion blur due to flow.

However, motion blur in a single X-ray image exposure due to the pipe rotation could be more significant. For a particle at the wall while flow induced motion blur is insignificant the motion blur due to rotation is appreciable whenever the particle is at an angle around $\alpha \approx 0$ and $180^{\circ}$ (see Fig. 10). However, when the particle is at an angle near 90 and $270^{\circ}$ there is very little motion blur in those projections. Hence a CT reconstruction even with the basic and fast FDK algorithm will still end up reconstructing a strong enough particle that its centroid can be localized with ease, as shown in Fig. 11. The simulation of this motion blur could further be refined in a planned follow-up paper dedicated to exploring motion and other artifacts specific to TXPTV in depth.

A second notable source of artifacts for CT, and also relevant to TXPTV, is motion within the domain during the a full $360^{\circ} \mathrm{CT}$ scan. For the present experiments motion due to flow during a $360^{\circ}$ rotation could be non-negligible and affect the particle reconstruction, and hence it is considered here succinctly and tentatively. For our datasets, a full rotation during which 200 projections were recorded took 2.9 seconds, and hence a $60 \mu \mathrm{m}$ particle moving at $5 . .50 \mu \mathrm{m} / \mathrm{sec}$ would move $0.24 . . .2 .42$ particle diameters. Such a motion is known to cause a "corkscrew-artifact," where, e.g., a sphere will take on the shape that is more twisted like a corkscrew, with distortion being more significant the more pronounced

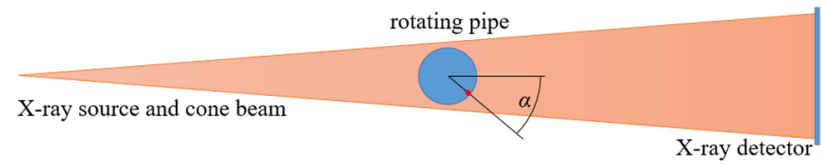

Fig. 10 Sketch of projection geometry showing a particle (red dot) on the pipe wall, where blur during exposure will be worst when particle is near $\alpha \approx 0$ and $180^{\circ}$. Sketch not to scale
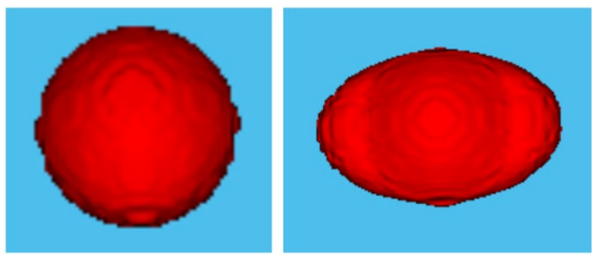

Fig. 11 Particle reconstructed based on synthetic data when (left) exposure had no motion blur, and (right) motion blur has been mimicked in synthetic data by having particle attenuation coefficient in pixels occupied by particle during exposure have value proportional to that due to the approximate average content of voxels during exposure. For case where at worst particle at $\alpha \approx 0^{\circ}$ moves six particle diameters due to pipe rotation the reduction effective attenuation is approximated as $\frac{1}{6}+\frac{5}{6}\left(\frac{1}{2}+\frac{1}{2} \cos 2[\alpha+\pi / 2]\right)$
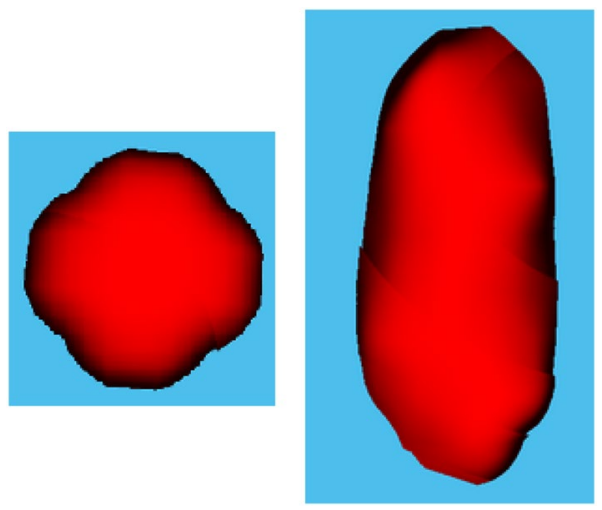

Fig. 12 Particle created with synthetic data simulating noise-free monochromatic scan data of $60 \mu \mathrm{m}$ homogeneous particles data when (left) no motion, and (right) two diameters of pure axial motion during the full $360^{\circ}$ scan $(2.9$ seconds). While particle shape becomes distorted, its centroid can sill be easily localized

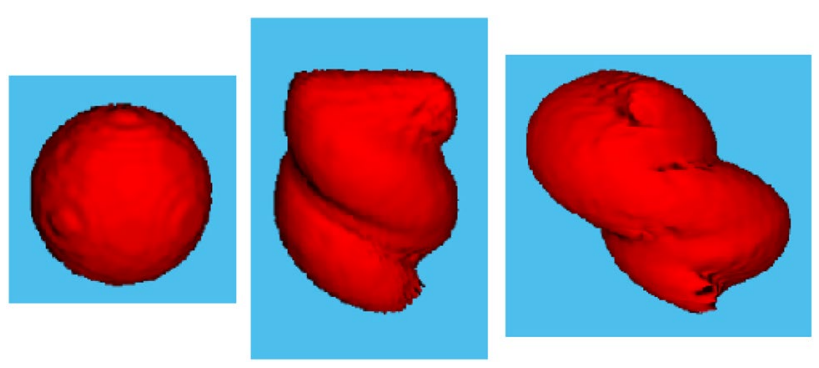

Fig. 13 Particle created with synthetic data by simulating noise-free monochromatic scan data of $400 \mu \mathrm{m}$ homogeneous particles when (left) no motion, (center) one diameter of pure axial motion and (right) one diameter of both axial and in-plane motion during the full $360^{\circ}$ scan (2.9 seconds). Not the "corkscrew"-shape becomes evident

the motion is. However, for TXPTV the key thing to note is that the centroid location of the reconstructed object remains nominally unaffected up to rather extreme artifacts.

Let us consider a CT reconstruction from synthetic 3D CT data of a sphere that moved during a CT scan. First in Fig. 12 we consider pure axial motion. For computational efficiency we simulate noise-free monochromatic scan data of $60 \mu \mathrm{m}$ homogeneous particles with SID, SOD and detector pixel pitch matching those used in the present study.

As the particle image in the present dataset and in previous example is only about 4 voxels wide it is difficult to make out much detail of the shape distortion. Hence in Fig. 13 to highlight shape distortion we consider a hypothetical particle that occupied 300x more voxels than the tracers used (i.e., $400 \mu \mathrm{m}, 7.6 \mathrm{x}$ larger diameter).

The above reconstructions were obtained using a CUDA implemented version of the FDK algorithm used in this paper for all datasets. If, however, we were to use more advances such as simultaneous iterative reconstruction 
technique (SIRT) reconstruction it is even easier to see how a particle, albeit distorted, yields usable data and particle's centroid is still easy to localize. Hence TXPTV is more forgiving of motion artifacts than CT of structures, as clearly the shape of a structure would be distorted unacceptably by even modest motion whilst even with simple reconstruction (FDK) locating a particle centroid can succeed for motion far exceeding one particle diameter.

In a planned follow-up study, we will further in-depth consider effect of motion artifacts for TXPTV, and how these artifacts can be minimized. The above consideration was idealized to illustrate simple points, but a dedicated study should additionally (at least to some degree) consider the effect of polychromatic spectrum, imperfect imager response, scatter, acquisition parameters, interframe blur, pre-processing steps, reconstruction algorithm, post-processing algorithm (as deformation shape is known localization can be done accounting for this in part), etc. However, such a full consideration of possible motion and other imaging artifacts of TXPTV will require significant time and resources and was beyond the scope of the present paper.

Supplementary Information The online version contains supplementary material available at https://doi.org/10.1007/s00348-021-03362-w.

Acknowledgements The first author gratefully acknowledges the support of NSF EAGER award \#1922877 program manager Ron Joslin and Shahab Shojaei-Zadeh. We also wish to thank Prof. Michael Riemer of UC Berkeley who generously provided use of his sieving equipment and even volunteered to sieve the tracer particles.

Open Access This article is licensed under a Creative Commons Attribution 4.0 International License, which permits use, sharing, adaptation, distribution and reproduction in any medium or format, as long as you give appropriate credit to the original author(s) and the source, provide a link to the Creative Commons licence, and indicate if changes were made. The images or other third party material in this article are included in the article's Creative Commons licence, unless indicated otherwise in a credit line to the material. If material is not included in the article's Creative Commons licence and your intended use is not permitted by statutory regulation or exceeds the permitted use, you will need to obtain permission directly from the copyright holder. To view a copy of this licence, visit http://creativecommons.org/licenses/by/4.0/.

\section{References}

Atkinson B, Brocklebank M, Card C, Smith J (1969) Low reynolds number developing flows. AIChE J 15(4):548-553

Attwood D, Sakdinawat A (2017) X-rays and extreme ultraviolet radiation: principles and applications. Cambridge University Press, Cambridge

Borgefors G (1984) Distance transformations in arbitrary dimensions. Computer Vis Gr, Image Process 27(3):321-345. https://doi.org/ 10.1016/0734-189X(84)90035-5

Buades A, Coll B, Morel JM (2005) A non-local algorithm for image denoising. In: IN CVPR, pp 60-65

Cheng NS (2008) Formula for the viscosity of a glycerol- water mixture. Ind Eng Chem Res 47(9):3285-3288
Dubsky S, Jamison R, Irvine S, Siu K, Hourigan K, Fouras A (2010) Computed tomographic X-ray velocimetry. Appl Phys Lett 96(2):023702

Espes E, Andersson T, Bjornsson F, Gratorp C, Hansson BA, Hemberg O, Johansson G, Kronstedt J, Otendal M, Tuohimaa T et al (2015) Liquid-metal-jet $\mathrm{x}$-ray tube technology and tomography applications. In: Digital Imaging XVIII, pp 7-11

Feldkamp LA, Davis LC, Kress JW (1984) Practical cone-beam algorithm. Josa a 1(6):612-619

Ganesh H, Mäkiharju SA, Ceccio SL (2016) Bubbly shock propagation as a mechanism for sheet-to-cloud transition of partial cavities. J Fluid Mech 802:37-78

Im KS, Fezzaa K, Wang Y, Liu X, Wang J, Lai MC (2007) Particle tracking velocimetry using fast $\mathrm{X}$-ray phase-contrast imaging. Appl Phys Lett 90(9):091919

Kak A, Slaney M (1988) Principles of computerized tomographic imaging ieee press. New York

Lee SJ, Kim GB (2003) X-ray particle image velocimetry for measuring quantitative flow information inside opaque objects. J Appl Phys 94(5):3620-3623

Lee SJ, Kim S (2005) Simultaneous measurement of size and velocity of microbubbles moving in an opaque tube using an $\mathrm{x}$-ray particle tracking velocimetry technique. Exper Fluids 39(3):492-497

Linden S, Wiegmann A, Hagen H (2015) The lir space partitioning system applied to the stokes equations. Gr Models 82:58-66. https:// doi.org/10.1016/j.gmod.2015.06.003

Liu Q, Zhao B, Santamarina J (2019) Particle migration and clogging in porous media: a convergent flow microfluidics study. J Geophys Res: Solid Earth 124(9):9495-9504

Mäkiharju SA, Gabillet C, Paik BG, Chang NA, Perlin M, Ceccio SL (2013) Time-resolved two-dimensional x-ray densitometry of a two-phase flow downstream of a ventilated cavity. Exp Fluids 54(7): $1-21$

Mäkiharju SA, Ganesh H, Ceccio SL (2017) The dynamics of partial cavity formation, shedding and the influence of dissolved and injected non-condensable gas. J Fluid Mech 829:420

Otsu N (1979) A threshold selection method from gray-level histograms. IEEE Trans Syst, Man, Cybern 9(1):62-66. https://doi. org/10.1109/TSMC. 1979.4310076

Parker JT, Mäkiharju SA (2021) Experimentally Validated Simulations of $50 \mu \mathrm{m} \mathrm{X-ray} \mathrm{PIV} \mathrm{Tracer} \mathrm{Particles.} \mathrm{arXiv} \mathrm{preprint} \mathrm{arXiv:}$ 210913470

Pearson K (1901) On lines and planes of closest fit to systems of points in space. J Sci 2:559. https://doi.org/10.1080/14786440109462720

Raffel M, Willert CE, Scarano F, Kähler CJ, Wereley ST, Kompenhans J (2018) Particle image velocimetry: a practical guide. Springer, New York

Smith L, Kolaas J, Jensen A, Sveen K (2018) Investigation of surface structures in two phase wavy pipe flow by utilizing X-ray tomography. Int J Multiph Flow 107:246-255

Vincent L, Soille P (1991) Watersheds in digital spaces: An efficient algorithm based on immersion simulations. IEEE Trans Pattern Anal Mach Intell 13:583-598

Volk A, Kähler CJ (2018) Density model for aqueous glycerol solutions. Exper Fluids 59(5):1-4

Yoon S, Mäkiharju SA, Fessler JA, Ceccio SL (2017) Image reconstruction for limited-angle electron beam $\mathrm{x}$-ray computed tomography with energy-integrating detectors for multiphase flows. IEEE Trans Comput Imag 4(1):112-124

Publisher's Note Springer Nature remains neutral with regard to jurisdictional claims in published maps and institutional affiliations. 


\section{Authors and Affiliations}

\section{Simo A. Mäkiharju ${ }^{1}\left[\right.$. Jan Dewanckele ${ }^{2} \cdot$ Marijn Boone $^{2} \cdot$ Christian Wagner $^{3} \cdot$ Andreas Griesser $^{3}$}

1 University of California, 6119 Etcheverry Hall, Berkeley,

3 Math2Market GmbH, Kaiserslautern, Germany CA 94720, USA

2 Tescan, Ghent, Belgium 